\title{
Increased Fetal Plasma Erythropoietin in Monochorionic Twin Pregnancies With Selective Intrauterine Growth Restriction and Abnormal Umbilical Artery Doppler
}

\author{
Yao-Lung Chang, ${ }^{1}$ An-Shine Chao, ${ }^{1}$ Hsiu-Huei Peng, ${ }^{1}$ Shuenn-Dyh Chang, ${ }^{1}$ Sheng-Yuan Su, ${ }^{1}$ \\ Kuan-Ju Chen, ${ }^{1}$ Po-Jen Cheng, ${ }^{1}$ and Tzu-Hao Wang ${ }^{1,2,3}$ \\ ${ }^{1}$ Department of Obstetrics and Gynecology, Chang Gung Memorial Hospital, Linkou, College of Medicine, Chang Gung \\ University, Taoyuan, Taiwan \\ ${ }^{2}$ School of Traditional Chinese Medicine, College of Medicine, Chang Gung University, Taoyuan, Taiwan \\ ${ }^{3}$ Genomic Medicine Research Core Laboratory (GMRCL), Chang Gung Memorial Hospital, Taoyuan, Taiwan
}

\begin{abstract}
Hypoxia is the primary stimulus for the production of erythropoietin (EPO) in both fetal and adult life. Here, we investigated fetal plasma EPO concentrations in monochorionic (MC) twin pregnancies with selective intrauterine growth restriction (sIUGR) and abnormal umbilical artery (UA) Doppler. We diagnosed sIUGR in presence of (1) birth-weight discordance $>20 \%$ and (2) either twin with a birth weight $<10$ th percentile. An abnormal UA Doppler was defined as a persistent absent-reverse end diastolic flow (AREDF). The intertwin EPO ratio was calculated as the plasma EPO level of the smaller (or small-for-gestational-age) twin divided by the EPO concentration of the larger (or appropriate-for-gestational-age (AGA)) twin. Thirty-two MC twin pairs were included. Of these, 17 pairs were normal twins (Group 1), seven pairs were twins with sIUGR without UA Doppler abnormalities (Group 2), and eight pairs were twins with sIUGR and UA Doppler abnormalities (Group 3). The highest EPO ratio was identified in Group $3(p<.001)$ but no significant differences were observed between Groups 1 and 2. Fetal hemoglobin levels did not differ significantly in the three groups, and fetal EPO concentration did not correlate with gestational age at birth. We conclude that fetal plasma EPO concentrations are selectively increased in MC twin pregnancies with sIUGR and abnormal UA Doppler, possibly as a result of uncompensated hypoxia.
\end{abstract}

Keywords: erythropoietin, monochorionic twin pregnancy, intrauterine growth restriction, placenta

Hypoxia is the primary stimulus for the production of EPO in both fetal and adult life (Teramo \& Widness, 2009). EPO is unable to cross the human placenta (Widness et al., 1995) and cannot undergo tissue storage for subsequent release. In this scenario, fetal plasma EPO levels closely reflect the balance between acute EPO production in the fetus and its subsequent clearance. The half-life of circulating EPO in the bloodstream is approximately 4-8 hours in adult life (Jelkmann, 1992) and 2-4 hours in newborn infants (Widness et al., 1996). Increased fetal plasma EPO levels are inversely correlated with UA $\mathrm{pH}$ and base excess at birth in pregnancies with growth restriction (Lemery et al., 1994). It has therefore been suggested that increased fetal plasma EPO concentrations can be a biomarker of chronic or subchronic fetal hypoxia (Teramo et al., 2004).

Fetal plasma EPO levels are higher in growth restricted fetuses (Doi et al., 1999; Jazayeri et al., 1999; Lemery et al., 1994). However, extrinsic factors (e.g., uterine contractions) can also influence its concentrations (Widness et al., 1984). Consequently, the exact relationship between plasma EPO concentrations and the occurrence of intrauterine growth restriction (IUGR) remains controversial because of potential confounders. sIUGR - defined as the presence of one twin with IUGR with the other twin being AGA - can be identified in 12-15\% of all MC twin pregnancies (Lewi et al., 2003). Because the two fetuses of a MC twin pregnancy with sIUGR share an identical genetic

RECEIVED 14 January 2016; ACCEPTED 17 March 2016. First published online 10 May 2016.

AdDRESS FOR CORRESPONDENCE: Tzu-Hao Wang, No.5, Fu-Shin Road, Gwei-Shan, Taoyuan, Taiwan. E-mail: knoxtn@cgmh.org.tw 
make-up, fetal plasma EPO concentrations of the AGA fetus can serve as an internal control for the corresponding levels detected in the sIUGR twin. In this setting, the detection of significant differences in fetal EPO concentrations in MC twin pregnancies with sIUGR (accompanied by the lack of such differences in MC twin pregnancies without sIUGR) would suggest a potential role of fetal plasma EPO in growth restriction.

Starting from these premises, we designed the current study to compare fetal plasma EPO concentrations in MC twin pairs categorized according to the presence of (1) sIUGR and (2) abnormal UA Doppler.

\section{Materials and Methods}

We analyzed consecutive MC twins delivered between February 2013 and September 2015 at the Chang Gung Memorial Hospital, Linkou Medical Center (a tertiary referral hospital in Taiwan). The study protocol was approved by the Institutional Review Board of the Chang Gung Medical Foundation (IRB \#101-4803A3). Patients who provided their informed consent and had cord blood successfully collected were included. Because uterine contractions may modify fetal plasma EPO levels (Teramo \& Widness, 2009), only MC twins delivered by cesarean section before the beginning of the labor were deemed eligible. Pregnancies with twin-twin transfusion syndrome (TTTS), twin anemiapolycythemia sequence (TAPS), and congenital, structural, or genetic malformations were excluded. The diagnosis of TTTS was based on the Quintero's criteria (Quintero et al., 1999). TAPS was diagnosed in presence of a postnatal intertwin hemoglobin difference $>8 \mathrm{~g} / \mathrm{dL}$ (Lopriore et al., 2010). All of the MC twin pregnancies included in the current study were identified by ultrasound during the first trimester or the early second trimester using the following criteria: (1) presence of a single placenta, (2) presence of a thin dividing membrane, and (3) lack of a twin peak sign. Monochorionicity was confirmed by obstetricians through the postpartum examination of the placenta (presence of a single placenta and occurrence intertwin anastomoses).

We diagnosed sIUGR in presence of (1) birth-weight discordance $>20 \%$ and (2) either twin with a birth weight $<10$ th percentile (according to a standard singleton pregnancy birth weight chart). Birth-weight discordance was calculated as the difference between the fetal weight of the larger and the smaller twins divided by the fetal weight of the larger twin, as follows: [body weight of the larger (AGA) twin - body weight of the smaller (sIUGR) twin] $\times 100 \%$ /(body weight of the larger twin). An abnormal UA Doppler was defined as a persistent AREDF. MC twins were categorized in three groups, as follows: normal MC twins (Group 1), twins with sIUGR without UA Doppler abnormalities (Group 2), and twins with sIUGR with UA Doppler abnormalities (Group 3). MC twin pregnancies in Groups 2 and 3 were complicated by sIUGR and birth-weight discordance was $>20 \%$ (Chang et al., 2008).
The umbilical cord of each baby was doubly clamped during cesarean section. After delivery and before removal of the placenta, surgeons on the operating table collected blood samples from the clamped cord at the placental side. Samples were subsequently sent to the Department of Clinical Pathology for EPO measurements. Fetal plasma EPO concentrations were quantified with a commercially available chemiluminescent immunometric assay (IMMULITE $^{\circledR} 2000$ systems, Siemens, Los Angeles, USA). The intertwin EPO ratio was defined as the plasma EPO levels of the smaller (or small-for-gestional-age) twin divided by the EPO concentration of the larger (or AGA) twin.

Data were analyzed with the SPSS 11.0 statistical software (SPSS Inc, Chicago, IL, USA). Intergroup comparisons of continuous variables in the three study groups were performed with one-way analysis of variance (ANOVA) followed by post-hoc Fisher's least significance difference (LSD) tests for multiple comparisons. Paired Student's $t$ tests were used to compare continuous data within each pair. The sex distribution between different pregnancy groups was examined with the $\chi^{2}$ test. The correlations between EPO ratios, gestational ages at birth, and intertwin birthweight discordance were analyzed with Pearson's correlation coefficients. Two-tailed $p$ values $<.05$ were considered statistically significant.

\section{Results}

Thirty-two MC twin pairs were included in the study (17 in Group 1, seven in Group 2, and eight in Group 3). MC twins in Group 3 were delivered significantly earlier (mean: 32.4 weeks) than those in Groups 1 and 2. The highest EPO ratio was identified in Group 3 twin pregnancies (Table 1).

Because of the insufficient cord blood quantity obtained from three fetuses with sIUGR and UA Doppler abnormalities, such cases were excluded from Group 3. Hemoglobin values did not differ significantly between the larger (appropriate-for-gestional-age) and smaller (smallfor-gestional-age) fetuses in all of the three study groups (Table 2).

The EPO ratio observed in Group 1 served as control for EPO ratios measured in Groups 2 and 3. The EPO ratio in Group 3 was significantly higher than those identified in Groups 1 and 2. However, no significant differences were evident between Groups 1 and 2 (Figure 1).

The EPO ratios showed a significant positive correlation with birth-weight discordance $(p<.001$; Figure 2$)$. An outlier characterized by a markedly high EPO ratio (4.92) was identified in Group 3 (Figure 2). This case showed the highest intertwin birth-weight discordance (54.9\%) observed in the current study. Specifically, the birth weight of the AGA twin was $1750 \mathrm{~g}$, whereas that of the sIUGR fetus was $790 \mathrm{~g}$.

Because gestational ages at birth were found to differ significantly in the three study groups, Pearson's correlation coefficients were used to investigate the association 
TABLE 1

Characteristics of the Three Groups of Monochorionic Twin Pregnancies

\begin{tabular}{lcccc}
\hline & Group $1(n=17)$ & Group $2(n=7)$ & Group $3(n=8)$ & $p$ value \\
\hline Gestational age of delivery (weeks) & $36.1 \pm 1.1$ & $34.0 \pm 1.6$ & $32.1 \pm 2.2$ & $<.001^{\#}(\mathrm{Group} 1>\mathrm{Group} 2>\mathrm{Group} 3)$ \\
Sex of the fetuses (male/female) & $11 / 6$ & $4 / 3$ & $3 / 5$ & $.441 *$ \\
Birth weight of the AGA (larger) twin (g) & $2,372 \pm 317$ & $2,085 \pm 395$ & $1,634 \pm 375$ & $.002^{\#}$ (Groups 1 and 2 $>$ Group 3) \\
Birth weight of the sIUGR (smaller) twin (g) & $2,189 \pm 308$ & $1,455 \pm 285$ & $891 \pm 122$ & $<.001(\mathrm{Group} 1>$ Group 2 $>$ Group3) \\
Birth weight discordance (\%) & $7.7 \pm 6.0$ & $29.9 \pm 7.9$ & $44.0 \pm 8.7$ & $<.001^{\#}$ (Group3 $>$ Group2 $>$ Group1) \\
EPO ratio & $1.0 \pm 0.18$ & $1.45 \pm 0.66$ & $2.53 \pm 1.1$ & $.004^{\#}$ (Group3 $>$ Groups 1 and 2)
\end{tabular}

Note: Group 1: normal MC twins; Group 2: twins with sIUGR without UA Doppler abnormalities; Group 3: twins with sIUGR and UA Doppler abnormalities. $M C=$ monochorionic; sIUGR = selective intrauterine growth restriction; $A G A=$ appropriate-for-gestational-age; $E P O=$ erythropoietin. Birth-weight discordance: [(body weight of the larger (AGA) twin - body weight of the smaller (sIUGR) twin/body weight of the larger twin] $\times$ $100 \%$.

\#One-way analysis of variance; $* \chi 2$ test.

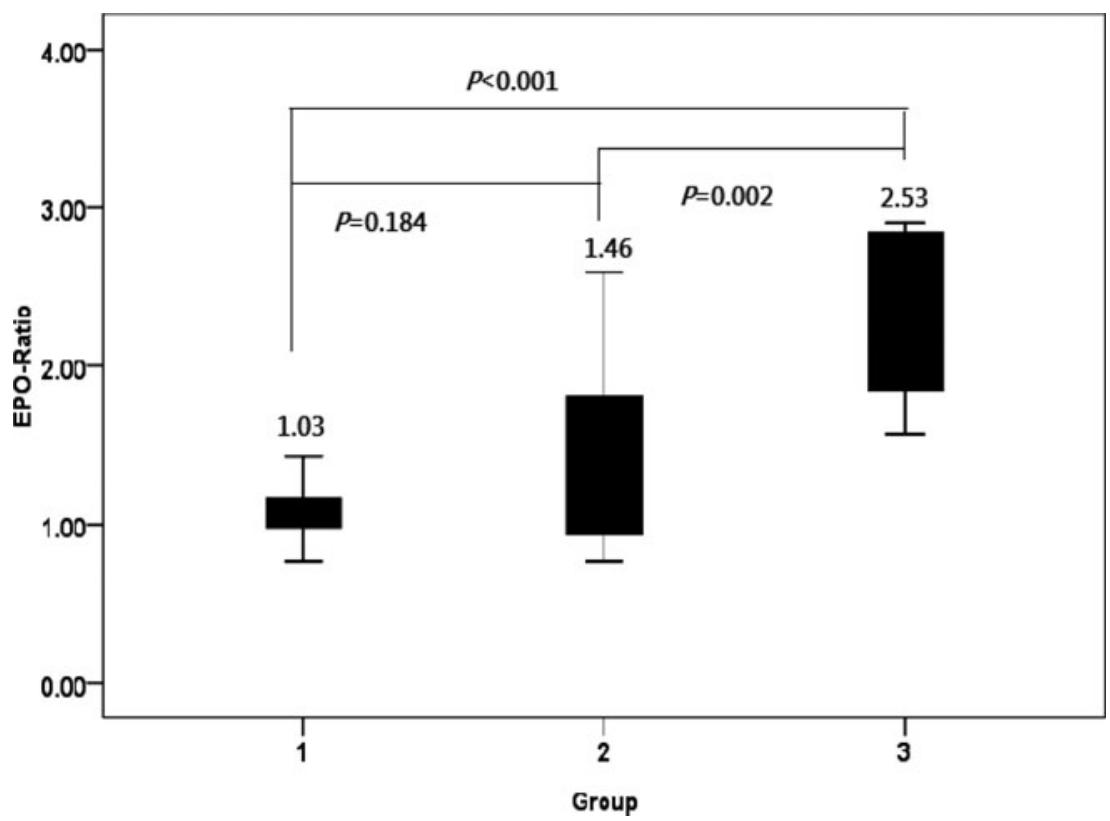

\section{FIGURE 1}

EPO ratios in the three groups of monochorionic twin pregnancies.

Note: The EPO ratio was significantly higher in Group 3 (one-way analysis of variance, $p<.001$ ), but no significant differences were detected between Groups 1 and 2 (post-hoc Fisher's least significance difference tests for multiple comparisons).

Group 1: normal MC twins; Group 2: twins with sIUGR without UA Doppler abnormalities; Group 3: twins with sIUGR and UA Doppler abnormalities.

$\mathrm{EPO}=$ erythropoietin; sIUGR = selective intrauterine growth restriction; $\mathrm{AGA}=$ appropriate-for-gestational-age; $\mathrm{UA}=$ umbilical artery . EPO ratio: [(EPO concentration of the smaller (sIUGR) twin/EPO concentration of the larger (AGA) twin]

between gestational age at delivery and fetal plasma EPO concentrations. No significant correlations were identified between gestational age at delivery and plasma EPO concentrations both in the smaller (sIUGR; $p=.427$ ) and larger (appropriate-for-gestional-age) fetuses ( $p=.898$; Figure 3 ).

\section{Discussion}

The main results of our study focusing on MC twin pregnancies indicate that fetal plasma EPO concentrations are higher in sIUGR fetuses only in presence of an abnormal UA Doppler. Because increased plasma EPO levels are
TABLE 2

Hemoglobin Values ( $g / d L)$ of Fetuses at Delivery in the Three Groups of Monochorionic Twin Pregnancies

\begin{tabular}{llll}
\hline & Larger (AGA) fetuses & Smaller (sIUGR) fetuses & p value \\
\hline Group 1 & $16.84(14.9-20.5)$ & $16.74(15.2-21.1)$ & $.714^{*}$ \\
Group 2 & $17.44(15.1-19.7)$ & $17.30(14.8-20.5)$ & $.788^{*}$ \\
Group 3 & $17.24(15.0-19.7)$ & $17.03(15.3-20.7)$ & $.698^{*}$ \\
\hline
\end{tabular}

Note: Data are expressed as means (ranges in parentheses). Group 1: normal MC twins; group 2: twins with sIUGR without UA Doppler abnormalities; group 3: twins with sIUGR and UA Doppler abnormalities. $M C=$ monochorionic; sIUGR = selective intrauterine growth restriction; AGA = appropriate-for-gestational-age.

*Paired sample Student's t test. 


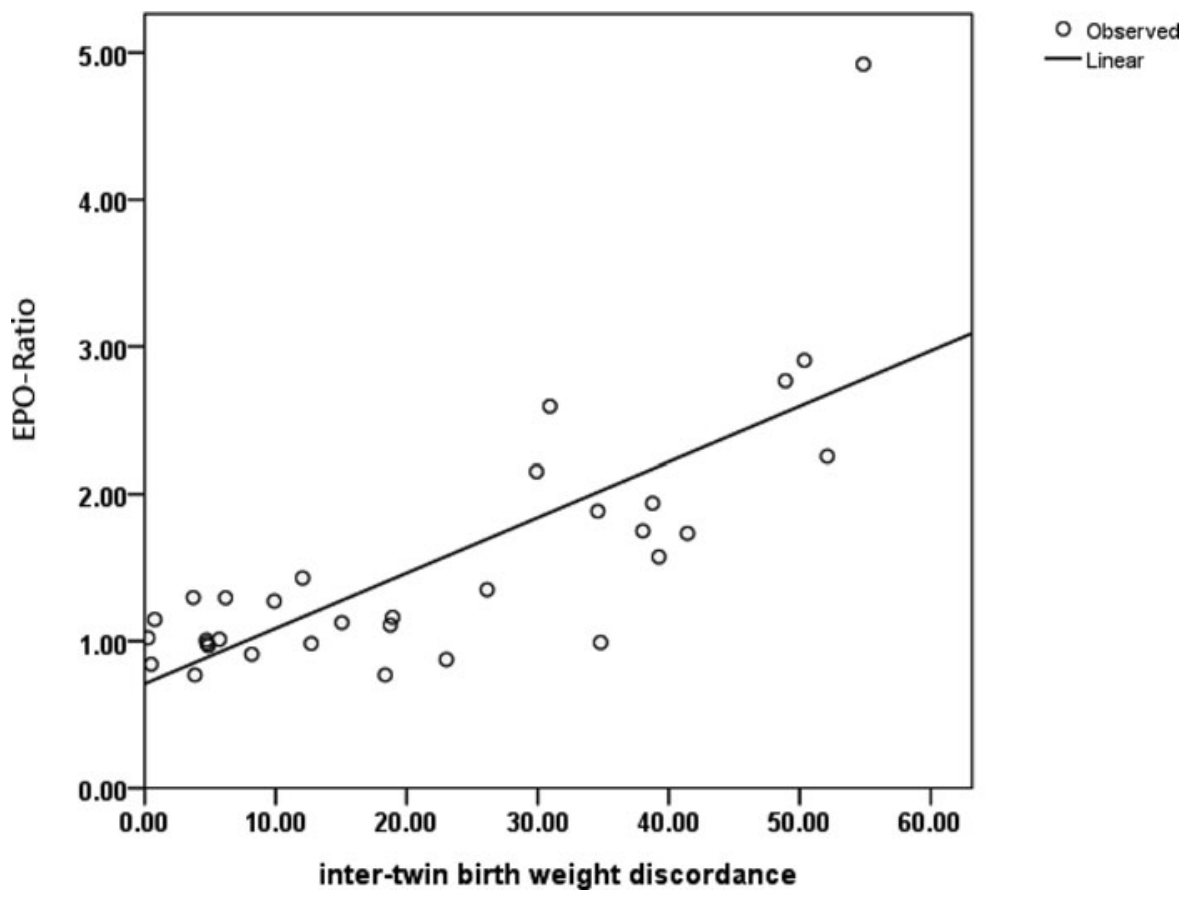

\section{FIGURE 2}

Scattergram and regression line depicting the relationship between the EPO ratio and intertwin birth-weight discordance.

Note: A significant positive correlation between the EPO ratio and intertwin birth-weight discordance was identified ( $p<.001$, Pearson's correlation analysis).

EPO = erythropoietin; sIUGR = selective intrauterine growth restriction; AGA = appropriate-for-gestational-age.

EPO ratio: [(EPO concentration of the smaller (sIUGR) twin/EPO concentration of the larger (AGA) twin].

Birth-weight discordance: [(body weight of the larger (AGA) twin - body weight of the smaller (sIUGR) twin/body weight of the larger twin] $\times 100 \%$.

(a)

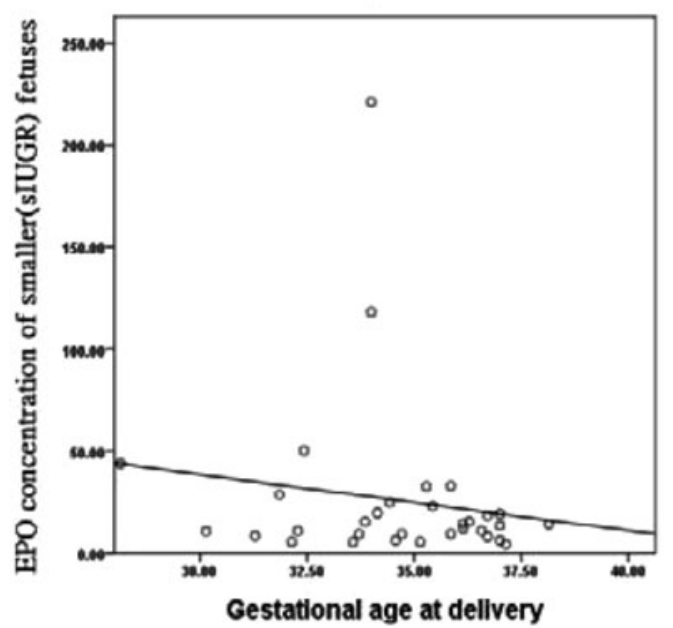

(b)

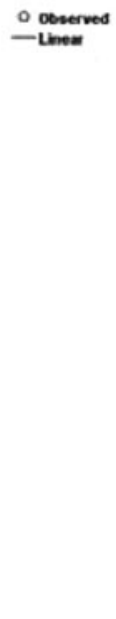

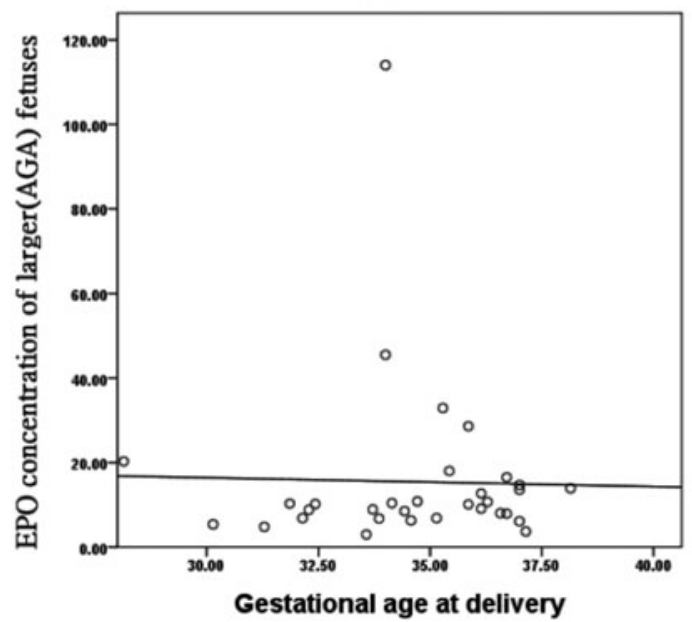

\section{FIGURE 3}

Scattergrams and regression lines depicting the relationships between fetal EPO concentrations and gestational age at delivery in sIUGR (panel a) and AGA (panel B) fetuses.

Note: No significant associations were identified between fetal EPO concentrations and gestational age at delivery both in sIUGR ( $p=.427$, Pearson's correlation analysis) and AGA ( $p=.898$, Pearson's correlation analysis) fetuses. EPO concentrations are given in $\mathrm{mIU} / \mathrm{mL}$, whereas gestational age at delivery is expressed in weeks.

EPO = erythropoietin; sIUGR = selective intrauterine growth restriction; AGA = appropriate-for-gestational-age. 
considered to reflect hypoxia (Teramo \& Widness, 2009), the current data support that the view that abnormal UA Doppler findings are linked to an impaired oxygen transport. Notably, we have previously shown that sIUGR twins with UA-AREDF have poorer perinatal outcomes than those with normal UA Doppler (Chang et al., 2009). Here, we have also shown that the severity of hypoxia (as reflected by the EPO ratio; Figure 1) is lower in Group 2 (sIUGR twins without UA Doppler abnormalities) than in Group 3 (sIUGR twins with UA Doppler abnormality). However, no significant differences in hemoglobin values were evident in the three study groups. Consequently, intertwin differences of the EPO ratio in Group 3 were not paralleled by alterations in hemoglobin levels. In our study, we identified an outlier with a markedly high EPO ratio (Figure 2). This case had an abnormal UA Doppler and was characterized by the highest birth-weight discordance observed in the current report. These findings suggest that the extent of hypoxia may exceed - and not necessarily parallel — intertwin birth-weight discordance in such extreme cases.

The lack of a significant correlation between gestational age at birth and fetal plasma EPO levels (Figure 3) is in line with a previous report showing that fetal plasma EPO concentrations do not vary according to gestational age throughout the latter half of normal human gestation (Teramo \& Widness, 2009). The results reported in Figure 3 do not support a major role for premature birth as a cause of the positive correlation identified between fetal plasma EPO levels and birth weight-discordance in MC twins (Figure 2).

Although MC twins (who share both an identical genetic make-up and the same maternal environment) represent an ideal model for studying the role of fetal plasma EPO in sIUGR, some limitations of the current study need to be acknowledged. Although TTTS cases were excluded, the potential presence of mild imbalances of blood flow between the twins through placental anastomoses cannot be ruled out completely. This potential confounder might have had an impact on the EPO ratios observed in MC twin pairs. Monozygotic dichorionic twins with sIUGR would represent a better model for investigating the relationships between fetal plasma EPO levels and growth restriction. Unfortunately, both their rarity and the need for specific tests for confirmation of zygosity assignment (e.g., short tandem repeat analysis or blood typing) have hampered this research field to date. The question as to whether different types of intertwin anastomoses may affect the EPO ratio remains open. Finally, the small sample size is a major caveat inherent in our study, only partly tempered by the use of an ideal research model (i.e., MC twins sharing identical genetic and environmental conditions). Under these circumstances, we cannot exclude that the non-significant differences in EPO ratios between Groups 1 and 2 could become significant with a larger sample size.
We conclude that fetal plasma EPO concentrations are selectively increased in MC twin pregnancies with sIUGR and abnormal UA Doppler. Notably, the EPO ratios showed a significant positive correlation with intertwin birth-weight discordance. Taken together, our results indicate that fetal EPO concentrations are higher in growth restricted fetuses with abnormal UA Doppler, which may reflect the presence of uncompensated hypoxia.

\section{References}

Chang, Y. L., Chang, S. D., Chao, A. S., Hsieh, P. C., Wang, C. N., \& Tseng, L. H. (2008). The individual fetal weight/estimated placental weight ratios in monochorionic twins with selective intrauterine growth restriction. Prenatal Diagnosis, 28, 217-221.

Chang, Y. L., Chang, S. D., Chao, A. S., Hsieh, P. C., Wang, C. N., \& Wang, T.H. (2009). Clinical outcome and placental territory ratio of monochorionic twin pregnancies and selective intrauterine growth restriction with different types of umbilical artery Doppler. Prenatal Diagnosis, 29, 253-256.

Doi, S., Osada, H., Seki, K., \& Sekiya, S. (1999). Relationship of amniotic fluid index and cord blood erythropoietin levels in small for and appropriate for gestational age fetuses. Obstetrics \& Gynecology, 94, 768-772.

Jazayeri, A., Tsibris, J. C., \& Spellacy, W. N. (1999). Fetal erythropoietin levels in growth-restricted and appropriately grown neonates with and without abnormal fetal heart rate tracings: A comparison with cord blood gases and Apgar scores. Journal of Perinatology, 19, 255-259.

Jelkmann, W. (1992). Erythropoietin: Structure, control of production, and function. Physiological Reviews, 72, 449489.

Lemery, D. J., Santolaya, J., Serre, A. F., Denoix, S., Besse, G. H., Vanlieferinghen, P. C., ... Jacquetin, B. (1994). Serum erythropoietin in small for gestational age fetuses. Biology of the Neonate, 65, 89-93.

Lewi, L., Van Schoubroeck, D., Gratacos, E., Witters, I., Timmerman, D., \& Deprest, J. (2003). Monochorionic diamniotic twins: Complications and management options. Current Opinion in Obstetric \& Gynecology, 15, 177-194.

Lopriore, E., Slaghekke, F., Oepkes, D., Middeldorp, J. M., Vandenbussche, F. P., \& Walther, F. J. (2010). Hematological characteristics in neonates with twin anemia-polycythemia sequence (TAPS). Prenatal Diagnosis, 30, 251255.

Quintero, R. A., Morales, W. J., Allen, M. H., Bornick, P. W., Johnson, P. K., \& Kruger, M. (1999). Staging of twin-twin transfusion syndrome. Journal of Perinatology, 19, 550-555.

Teramo, K. A., Hiilesmaa, V. K., Schwartz, R., Clemons, G. K., \& Widness, J. A. (2004). Amniotic fluid and cord plasma erythropoietin levels in pregnancies complicated by preeclampsia, pregnancy-induced hypertension and chronic hypertension. Journal of Perinatal Medicine, 32, 240-247. 
Teramo, K. A., \& Widness, J. A. (2009). Increased fetal plasma and amniotic fluid erythropoietin concentrations: Markers of intrauterine hypoxia. Neonatology, 95, 105116

Widness, J. A., Clemons, G. K., Garcia, J. F., Oh, W., \& Schwartz, R. (1984). Increased immunoreactive erythropoietin in cord serum after labor. American Journal of Obstetrics and Gynecology, 148, 194-197.
Widness, J. A., Schmidt, R. L., \& Sawyer, S. T. (1995). Erythropoietin transplacental passage - Review of animal studies. Journal of Perinatal Medicine, 23, 61-70.

Widness, J. A., Veng-Pedersen, P., Peters, C., Pereira, L. M., Schmidt, R. L., \& Lowe, L. S. (1996). Erythropoietin pharmacokinetics in premature infants: Developmental, nonlinearity, and treatment effects. Journal ofApplied Physiology (1985), 80, 140-148. 